\title{
Computing the Ehrhart Polynomial of a Convex Lattice Polytope*
}

\author{
A. I. Barvinok \\ MSI, 409 College Avenue, Ithaca, NY 14850, USA \\ barvinok@msiadmin.cit.cornell.edu
}

\begin{abstract}
We prove that computation of any fixed number of highest coefficients of the Ehrhart polynomial of an integral polytope can be reduced in polynomial time to computation of the volumes of faces.
\end{abstract}

\section{Introduction}

The problem of counting integral points in polyhedra has been of interest for a long time. It is known that generally this problem is \#P-hard. Even if we want to count integral points approximately with an exponentially large error, the problem still remains NP-hard [3]. In a recent paper [2] the author showed that if the dimension of a polytope is fixed then integral points in the polytope can be counted in polynomial time. In this paper we apply the technique of [2] further to deal with polytopes of varying dimension.

Let $\mathbb{Z}^{d} \subset \mathbb{R}^{d}$ be the standard integral lattice and let $P \subset \mathbb{R}^{d}$ be a polytope. We assume that $P$ is integral, namely, its vertices belong to $\mathbb{Z}^{d}$. For $m \in \mathbb{N}$ we put $m \cdot P=\{m \cdot x: x \in P\}$. Then the number of integral points in $m \cdot P$ is a polynomial in $m \in \mathbb{N}$, called the Ehrhart polynomial (see, for example, Chapter 4 of [12]). So

$$
\#\left\{m \cdot P \cap \mathbb{Z}^{d}\right\}=\sum_{j=0}^{d} e_{j}(P) \cdot m^{j} .
$$

In this paper we study the coefficients $e_{j}(P)$ from the computational complexity point of view.

\footnotetext{
* This research was supported by the United States Army Research Office through the Army Center of Excellence for Symbolic Methods in Algorithmic Mathematics (ACSyAM), Mathematical Sciences Institute of Cornell University, Contract DAAL03-91-C-0027.
} 
These coefficients naturally appear in many formulas of enumerative combinatorices (see, for example, [12]), therefore it might be interesting to compute them efficiently. It is well known that $e_{d}(P)$ is equal to the volume of $P$. The next coefficient $e_{d-1}(P)$ is half of the "surface area" of $P$, that is

$$
e_{d-1}(P)=\frac{1}{2} \cdot \sum_{G} \operatorname{vol}_{d-1}(G),
$$

where $G$ ranges over all facets of $P$ and the volume of a facet is measured intrinsically with respect to the lattice $\mathbb{Z}^{d} \cap A_{G}$, where $A_{G}$ is the affine hull of $G$ [9]. These observations show that computation of the two highest coefficients reduces to computation of the volumes of faces. From the results of [11] it follows that computation of $e_{d-2}(P)$ can also be reduced in polynomial time to computation of the volumes of faces ([11] does not deal with computational complexity but it contains an explicit expression of $e_{d-2}(P)$ in terms of the volumes and Dedekind sums; these sums, however, are known to be polynomially computable). We also know that $e_{0}(P)=1$ for an arbitrary polytope $P$ [9]. In this paper we prove that computation of any fixed number of the highest coefficients of the Ehrhart polynomial can be reduced to volume computation in polynomial time.

Let us assume that a $d$-dimensional integral polytope $P \subset \mathbb{R}^{d}$ is given by the set of linear inequalities

$$
P=\left\{x \in \mathbb{R}^{d}:\left\langle l_{i}, x\right\rangle \leq a_{i}, i=1, \ldots, m\right\},
$$

where $l_{i} \in \mathbb{Z}^{d}$ and $a_{i} \in \mathbb{Z}$ for all $i$. Here $\langle\cdot, \cdot\rangle$ denotes the standard inner product in $\mathbb{R}^{d}$. We use the standard notion of input size (see, for example, [6]). Thus for the polytope $P$ given by (1.1) we have

$$
\text { size } P=O\left(d \cdot \sum_{i=1}^{m}\left(1+\log \left(\left\|l_{i}\right\|+1\right)+\log \left(\left|a_{i}\right|+1\right)\right)\right)
$$

where $\|\cdot\|$ denotes the $L_{\infty}$ norm in $\mathbb{R}^{d}$. Let us introduce the

\section{Volume Computation Oracle.}

Input: a polytope $P$ given as in (1.1);

a set of indices $I \subset\{1, \ldots, m\}$ of cardinality $s$.

Output:

$$
\begin{cases}\operatorname{vol}_{d-s} F & \text { if the set } F=\left\{x \in P:\left\langle l_{i}, x\right\rangle=a_{i}, i \in I\right\} \\ & \text { is a }(d-s) \text {-dimensional face of } P, \\ & \text { otherwise. }\end{cases}
$$

Again, we measure the volume of a face $F$ intrinsically. Namely, let $A_{F}$ be the affine hull of $F$. Choosing a point from $\mathbb{Z}^{d} \cap A_{F}$ as the origin, let us consider $A_{F}$ as a linear space. Then $\mathbb{Z}^{d} \cap A_{F}$ is a lattice and we scale the volume so that the 
parallelopiped spanned by a basis of this lattice has volume 1 . Our main result is the following:

(1.2) Theorem. Let us fix $k \in \mathbb{N}$. Then, for any integral polytope $P$ given by (1.1), the coefficient $e_{d-k}(P)$ can be computed using $O\left(m^{k}\right)$ calls for the Volume Computation Oracle and a number of arithmetic operations which is polynomial in size $P$. The size of each number involved in the algorithm is bounded by a polynomial in size $P$.

The problem of volume computation is \# $P$-hard [4]. Moreover, the size of the volume is not necessarily bounded by a polynomial in the size of the polytope [8]. However, if we restrict ourselves to a class of integral polytopes, then the size of the volume of any face of such a polytope is bounded by a polynomial in the input size (since it is a rational number whose denominator is at most $d$ !). Therefore our Oracle is well defined in the context of Theorem 1.2. Although introduction of the Volume Computation Oracle which could solve \#P-complete problems seems to be too strong an assumption, it is unavoidable. Already computation of the highest coefficient of the Ehrhart polynomial reduces to volume computation. We note that volume computation generally seems to be somewhat easier than counting integral points. For example, the volume of a simplex can be computed by the explicit formula in the straightforward way whereas integral points counting is far less obvious. Theorem 1.2, however, implies the following result.

(1.3) Corollary. Let us fix $k \in \mathbb{N}$. For any given integral simplex $\Delta \subset \mathbb{R}^{d}$ the coefficient $e_{d-k}(\Delta)$ can be computed in polynomial time.

Theorem 1.2 implies, in particular, that integral points in an integral polytope can be counted in polynomial time if the dimension $d$ is fixed. This result is already contained in [2]. However, the present algorithm gives a better estimate of complexity. For a $d$-dimensional simplex $\Delta$ we get $(d \cdot \text { size } \Delta)^{o(d)}$ rather than (size $\Delta)^{O\left(d^{2}\right)}$ from [2].

In the first version of this paper [1] a weaker result was obtained by using a sort of "harmonic analysis on polytopes." The present paper uses a completely different approach based on some identities from [10]. We describe Morelli's identities in Section 2. To show that these identities lead to efficient algorithms we apply a technique of "cone decompositions" developed earlier in [2]. We describe this technique in Section 3. A new ingredient is an effective construction of a "generic subspace" required for Morelli's identities. This construction is described in Section 4. In Section 5 we prove Theorem 1.2 and give some estimates for the complexity of counting integral points in a simplex.

(1.4) Definitions and Notation. By co $S$ we denote the conic hull of the set $S \subset \mathbb{R}^{d}$. Thus

$$
\operatorname{co} S=\left\{x=\sum_{i} \lambda_{i} \cdot s_{i}: \lambda_{i} \geq 0, s_{i} \in S \text { for all } i\right\}
$$


By \#S we denote the cardinality of the set $S$. A polyhedral cone is a conic hull of finitely many integral vectors from $\mathbb{R}^{d}$. If $K=\operatorname{co}\left\{u_{1}, \ldots, u_{m}\right\} \subset \mathbb{R}^{d}$, where $u_{i} \in \mathbb{R}^{d}$ for $i=1, \ldots, m$, then we say that the cone $K$ is generated by $u_{1}, \ldots, u_{m}$ and call the vectors $u_{1}, \ldots, u_{m}$ generators of $K$. In what follows we consider only rational cones, that is, the cones generated by integral vectors. Similarly, a subspace is called rational if it is generated by integral vectors. We assume that the cone is given by its generators. A cone $K=\operatorname{co}\left\{u_{1}, \ldots, u_{m}\right\}$ is simple if $u_{1}, \ldots, u_{m}$ are linearly independent. By Lin $S$ we denote the linear hull of $S \subset \mathbb{R}^{d}$. A discrete additive subgroup $\Lambda \subset \mathbb{R}^{d}$ is called a lattice. Each lattice $\Lambda$ has a basis, that is, a set of linearly independent vectors $u_{1}, \ldots, u_{m} \in \Lambda$ such that

$$
\Lambda=\left\{x=\sum_{i=1}^{m} \lambda_{i} \cdot u_{i}: \lambda_{i} \in \mathbb{Z}\right\}
$$

Then $m$ is called the rank of $\Lambda$. The volume of the parallelopiped spanned by a basis of $\Lambda$ is called the determinant of the lattice $\Lambda$. Thus

$$
\operatorname{det} \Lambda=\left|u_{1} \wedge \cdots \wedge u_{m}\right|
$$

A simple cone $K=\operatorname{co}\left\{u_{1}, \ldots, u_{m}\right\}$ is called primitive if $u_{1}, \ldots, u_{m}$ is a basis of the lattice $\Lambda=$ Lin $K \cap \mathbb{Z}^{d}$. Then $u_{1}, \ldots, u_{m}$ are called primitive generators of $K$.

As a general reference to lattice algorithms we use [6].

\section{Morelli's Formulas}

In this section we briefly describe some results of [10]. Morelli's formulas provide an expression

$$
e_{d-k}(P)=\sum_{F} \operatorname{vol}_{d-k}(F) \cdot \mu(P, F)
$$

where $F$ ranges over the set of all $(d-k)$-dimensional faces of $P$ and $\mu(P, F)$ is a certain function which is determined by the supporting cone of $P$ at $F$. Actually, $\mu$ is an additive measure on rational polyhedral cones in $\mathbb{R}^{d}$. We describe this measure in detail.

Let us consider the set $\mathscr{C}_{k}$ of all $k$-dimensional rational polyhedral cones $K$ in $\mathbb{R}^{d}$. Morelli defines a measure $\mu$ on $\mathscr{C}_{k}$. For $K \in \mathscr{C}_{k}$, the value of $\mu(K)$ is a rational real-valued function on the Grassmanian $G_{d-k+1}\left(\mathbb{R}^{d}\right)$ of all $(d-k+1)$-dimensional subspaces in $\mathbb{R}^{d}$. If $G \in \mathbf{G}_{d-k+1}\left(\mathbb{R}^{d}\right)$ is a regular point of the function $\mu(K)$, then by $\mu_{G}(K)$ we denote the real number that is the value of the function $\mu(K)$ at the point $G$. Before we describe this measure we recall one useful notion. 
(2.1) Todd Polynomial. Todd polynomial $\operatorname{td}_{k}\left(x_{1}, \ldots, x_{d}\right)$ is defined as the coefficient of $t^{k}$ in the following expansion:

$$
\prod_{i=1}^{d} \frac{t \cdot x_{i}}{1-\exp \left\{-t \cdot x_{i}\right\}}=\sum_{k=0}^{+\infty} t^{k} \cdot \operatorname{td}_{k}\left(x_{1}, \ldots, x_{d}\right) .
$$

So, $\operatorname{td}_{k}\left(x_{1}, \ldots, x_{d}\right)$ is a symmetric homogeneous polynomial with rational coefficients of degree $k$. In Section 5 we need the following simple result.

(2.1.2) Lemma. For any given $d, k \in \mathbb{N}$ and for any rational nonzero numbers $x_{1}, \ldots, x_{d}$ the value of $\operatorname{td}_{k}\left(x_{1}, \ldots, x_{d}\right)$ can be computed in time which is polynomial in $d, k$, and the input size of $x_{1}, \ldots, x_{d}$.

Proof. For given $x_{1}, \ldots, x_{d}$ the value of $\operatorname{td}_{k}\left(x_{1}, \ldots, x_{d}\right)$ is equal to the $k$ th coefficient of the Taylor expansion of the univariate function from the left-hand side of (2.1.1). Replacing $\exp \left\{-t \cdot x_{i}\right\}$ by the first $k+1$ terms of its Taylor expansion we compute the desired value in the straightforward way.

First we define the function $\mu(K)$ for a primitive cone $K$.

(2.2) Primitive Cones. Let $K=\operatorname{co}\left\{u_{1}, \ldots, u_{k}\right\} \subset \mathbb{R}^{d}$ be a primitive cone with the primitive generators $u_{1}, \ldots, u_{k} \in \mathbb{Z}^{d}$. Let

$$
K^{*}=\left\{x \in \mathbb{R}^{d}:\langle x, y\rangle \geq 0 \text { for all } y \in K\right\} \subset \mathbb{R}^{d}
$$

be the conjugate cone. The cone $K^{*}$ contains a $(d-k)$-dimensional subspace

$$
V=\left\{x \in \mathbb{R}^{d}:\langle x, y\rangle=0 \text { for all } y \in K\right\}
$$

For $s=1, \ldots, k$ let us define a linear $(d-k+1)$-dimensional subspace $E_{s} \subset \mathbb{R}^{d}$ :

$$
E_{s}=\left\{x \in \mathbb{R}^{d}:\left\langle x, u_{j}\right\rangle=0 \text { for } j=1, \ldots, s-1, s+1, \ldots, k\right\}
$$

Let us choose a basis $x_{1}, \ldots, x_{d-k}$ of $V$. For each $s=1, \ldots, k$ we construct an oriented basis $B_{s}=\left(b_{1}^{s}, \ldots, b_{d-k+1}^{s}\right)$ of the $(d-k+1)$-dimensional lattice $E_{s} \cap \mathbb{Z}^{d}$. We assume that the orientation of $B_{s}$ is the same as that of the basis $x_{1}, \ldots, x_{d-k}, \bar{u}_{s}$, where $\bar{u}_{s}$ is the orthogonal projection of $u_{s}$ onto $E_{s}$.

Let us choose vectors $g_{1}, \ldots, g_{d-k+1} \in \mathbb{R}^{d}$. For $s=1, \ldots, k$ we define a $(d-k+1) \times(d-k+1)$ matrix $M^{s}$ as follows:

$$
M^{s}(i, j)=\left\langle b_{i}^{s}, g_{j}\right\rangle
$$


for $i, j=1, \ldots, d-k+1$. Put $f_{s}=\operatorname{det} M^{s}$. Let us put

$$
\mu_{g_{1}, \ldots, g_{k}}(K)=\frac{\operatorname{td}_{k}\left(f_{1}, \ldots, f_{k}\right)}{f_{1} \cdots f_{k}}
$$

Thus $\mu_{g_{1}, \ldots, g_{k}}(K)$ is a rational function in $g_{1}, \ldots, g_{d-k+1}$. Formally, $\mu$ depends also on the choice of $B_{s}$. However, since $\mathrm{td}_{k}$ is a homogeneous polynomial of degree $k$, we observe that the value of $\mu_{g_{1}, \ldots, g_{k}}(K)$ actually depends on the linear subspace $G \subset \mathbb{R}^{d}$ generated by $g_{1}, \ldots, g_{d-k+1}$ only. Thus for $G \in \mathbf{G}_{d-k+1}\left(\mathbb{R}^{d}\right)$ we put $\mu_{G}(K)=\mu_{g_{1}, \ldots, g_{d-k+1}}(K)$ where $g_{1}, \ldots, g_{d-k+1}$ is a basis of $G$. We observe that a "generic" $(d-k+1)$-dimensional subspace $G$ is a regular point of the function $\mu(K)$.

Morelli shows that $\mu$ is an additive measure.

(2.3) Theorem [10]. Let $K \subset \mathbb{R}^{d}$ be a $k$-dimensional rational cone. Let

$$
K=\bigcup_{i \in I_{1}} K_{i}
$$

and

$$
K=\bigcup_{i \in I_{2}} K_{i}
$$

be decompositions of the cone $K$ into the union of finitely many primitive $k$ dimensional cones $K_{i}, i \in I_{1}$ (resp. $K_{i}, i \in I_{2}$ ), with pairwise disjoint interiors. Then

$$
\sum_{i \in I_{1}} \mu\left(K_{i}\right)=\sum_{i \in I_{2}} \mu\left(K_{i}\right)
$$

as rational functions on the Gassmanian $\mathbf{G}_{d-k+1}\left(\mathbb{R}^{d}\right)$ of $(d-k+1)$-dimensional subspaces in $\mathbb{R}^{d}$.

Since every rational polyhedral $K$ cone can be decomposed into the union of finitely many primitive cones $K_{i}$ with pairwise disjoint interiors (see, for example, Section 2.6 of [5]) we can correctly define

$$
\mu(K)=\sum_{i \in I} \mu\left(K_{i}\right)
$$

Thus $\mu(K)$ is a rational function in $G \in \mathbf{G}_{d-k+1}\left(\mathbb{R}^{d}\right)$.

(2.4) Definition. Let $P \subset \mathbb{R}^{d}$ be a convex $d$-dimensional polytope. For a $(d-k)$ dimensional face $F \subset P$ of $P$ we define the supporting cone $K(F, P)$ as the cone of 
feasible directions. Let $y$ be an arbitrary point in the relative interior of the face $F$. We put $K(F, P)=\left\{x \in \mathbb{R}^{d}: y+\varepsilon \cdot x \in P\right.$ for all sufficiently small $\left.\varepsilon>0\right\}$. By $K^{*}(F, P)$ we denote the conjugate cone. Thus $\operatorname{dim} K^{*}(F, P)=k$.

(2.5) Theorem [10]. Let $P \subset \mathbb{R}^{d}$ be an integral polytope. Then

$$
e_{d-k}(P)=\sum_{F} \operatorname{vol}_{d-k}(F) \cdot \mu\left(K^{*}(F, P)\right)
$$

where the sum is taken over all $(d-k)$-dimensional faces $F$ of $P$. Thus the right-hand side is a constant function in $G \in \mathbf{G}_{d-k+1}\left(\mathbb{R}^{d}\right)$ whose value is equal to $e_{d-k}(P)$.

\section{Decomposition of Rational Cones}

In this section we show that any rational cone of a fixed dimension can be represented in polynomial time as a "linear combination" of primitive cones. For a set $S \subset \mathbb{R}^{d}$ by $\chi_{S}: \mathbb{R}^{d} \rightarrow\{0,1\}$ we denote the characteristic function of $S$, that is,

$$
\chi_{S}(x)= \begin{cases}1 & \text { if } x \in S \\ 0 & \text { otherwise }\end{cases}
$$

(3.1) Definition. Let $K, K_{i}, i \in I$, be a finite family of $k$-dimensional polyhedral cones in $\mathbb{R}^{d}$ and let $\varepsilon_{i}, i \in I$, be a family of integral numbers. We write

$$
K=\sum_{i \in I} \varepsilon_{i} \cdot K_{i}
$$

if and only if the identity

$$
\chi_{K}=\sum_{i \in I} \varepsilon_{i} \cdot \chi_{K_{i}}
$$

holds for all $x \in \mathbb{R}^{d}$ except possibly a finite union of rational $(k-1)$-dimensional subspaces.

Thus, if $K=\bigcup_{i \in I} K_{i}$, where $K_{i}, i \in I$, are $k$-dimensional rational cones with pairwise disjoint interors, we have that $K=\sum_{i \in I} K_{i}$. The following result is the standard corollary of Theorem 2.3 .

(3.2) Lemma. Let

$$
K=\sum_{i \in I} \varepsilon_{i} \cdot K_{i}
$$


be a representation of a $k$-dimensional rational polyhedral cone $K \subset \mathbb{R}^{d}$ into a linear combination of primitive $k$-dimensional cones $K_{i}, i \in I$. Then

$$
\mu(K)=\sum_{i \in I} \varepsilon_{i} \cdot \mu\left(K_{i}\right)
$$

Proof. Let $\Gamma_{j}, j \in J$, be a finite (possibly empty) union of ( $\left.k-1\right)$-dimensional subspaces from Definition 3.1. Let us subdivide all possible $k$-dimensional intersections $(K \cap) \bigcap_{i \in I_{i}} K_{i}, I_{1} \subset I$, into primitive $k$-dimensional cones $K_{\alpha}, \alpha \in A$, with pairwise disjoint interiors so that $\Gamma_{j}$ does not intersect the interior of $K_{\alpha}$ for all $j$ and $\alpha$. Thus each cone can be represented as a union $K=\bigcup_{\alpha \in A_{0}} K_{\alpha}, K_{i}=\bigcup_{\alpha \in A_{i}} K_{\alpha}$, so that, for any $\alpha \in A$,

$$
\sum_{i: \alpha \in A_{i}} \varepsilon_{i}= \begin{cases}1 & \text { if } \alpha \in A_{0} \\ 0 & \text { otherwise. }\end{cases}
$$

The proof then follows by Theorem 2.3 .

It is well known that a $k$-dimensional polyhedral cone $K \subset \mathbb{R}^{d}$ can be represented as a union of simple cones $K_{i}$ with pairwise disjoint interiors. If $k$ is fixed, then this can be done in polynomial time. Our aim is to show that if $k$ is fixed, then a simple rational $k$-dimensional cone can be represented as a linear combination of primitive cones in polynomial time. Here we basically follow [2], although we present a somewhat weaker construction since we do not care about points in a $(k-1)$-dimensional set.

We discuss the input size of our problem. For a simple cone $K=\operatorname{co}\left\{u_{1}, \ldots, u_{k}\right\}$, where $u_{i} \in \mathbb{Z}^{d}$, we have size $K=O\left(d \cdot \sum_{i=1}^{d}\left(1+\log \left(\left\|u_{i}\right\|+1\right)\right)\right)$, where $\|\cdot\|$ is the $L_{\infty}$ norm on $\mathbb{R}^{d}$.

(3.3) Definition. Let $K=\operatorname{co}\left\{u_{1}, \ldots, u_{k}\right\}$ be a simple $k$-dimensional cone in $\mathbb{R}^{d}$ given by its generators $u_{1}, \ldots, u_{k} \in \mathbb{Z}^{d}$. Let us put $\Lambda=\operatorname{Lin} K \cap \mathbb{Z}^{d}$. Thus $\Lambda$ is a $k$-dimensional lattice. By Ind $K$ we denote the index of the sublattice generated by the vectors $u_{1}, \ldots, u_{k}$ in the lattice $\Lambda$. In other words,

$$
\text { Ind } K=\frac{\left|u_{1} \wedge \cdots \wedge u_{k}\right|}{\operatorname{det} \Lambda}
$$

where $\left|u_{1} \wedge \cdots \wedge u_{k}\right|$ denotes the $k$-volume of the parallelopiped spanned by $u_{1}, \ldots, u_{k}$.

We conclude that $K$ is primitive with the primitive generators $u_{1}, \ldots, u_{k}$ if and only if Ind $K=1$. Besides, Ind $K$ is polynomially computable, and therefore the value of $\log$ Ind $K$ is bounded by a polynomial in the input size of the cone $K$. Our algorithm is based on successive reduction of Ind $K$. 
(3.4) Lemma (see [2]). Let us fix $k \in \mathbb{N}$. Then a polynomial-time algorithm exists which, for any $d \in \mathbb{N}$, for any rational simple $k$-dimensional cone $K \subset \mathbb{R}^{d}$, computes $k$-dimensional simple cones $K_{i}, i \in I, \# I \leq k$, and numbers $\delta_{i} \in\{+1,-1\}$ such that

$$
K=\sum_{i \in I} \delta_{i} \cdot K_{i}
$$

$$
\text { Ind } K_{i} \leq(\operatorname{Ind} K)^{(k-1) / k} \quad \text { for all } i \in I \text {, }
$$

$$
\text { size } K_{i} \leq \text { size } K+O\left(d^{2}\right) \quad \text { for all } i \in I \text {. }
$$

Proof. Let us consider the parallelopiped

$$
\Psi=\left\{x=\sum_{i=1}^{k} \alpha_{i} \cdot u_{i}:\left|\alpha_{i}\right| \leq(\operatorname{Ind} K)^{-1 / k}\right\}
$$

Thus $\Psi$ is a centrally symmetric convex body whose $k$-dimensional volume is equal to $2^{k} \cdot \operatorname{det} \Lambda$, where $\Lambda=\mathbb{Z}^{d} \cap \operatorname{Lin} K$. Therefore by Minkowski's theorem (see, for example, [6]) it contains a nonzero lattice vector $w \in \Lambda$. Choosing, if necessary, $-w$ instead of $w$ we ensure that $w, u_{1}, \ldots, u_{k}$ belong to certain open subspace in $\mathbb{R}^{d}$. Let us denote

$I=\left\{i \in[1: k]:\right.$ the vectors $u_{1}, \ldots, u_{i-1}, w, u_{i+1}, \ldots, u_{k}$ are linearly independent $\}$.

For $i \in I$ let us put $K_{i}=\operatorname{co}\left\{u_{1}, \ldots, u_{i-1}, w, u_{i+1}, \ldots, u_{k}\right\}$. We are going to prove that $K_{i}$ satisfy (3.4.1)-(3.4.3). Let us put

$$
\begin{aligned}
I_{+}= & \left\{i \in I: \text { the bases }\left(u_{1}, \ldots, u_{i-1}, w, u_{i+1}, \ldots, u_{k}\right) \text { and }\left(u_{1}, \ldots, u_{i-1}, u_{i}, u_{i+1}, u_{k}\right)\right. \\
& \text { have the same orientation }\}
\end{aligned}
$$

and

$$
I_{-}=I \backslash I_{+}
$$

Then we put

$$
\delta_{i}=\left\{\begin{array}{lll}
1 & \text { if } & i \in I_{+} \\
-1 & \text { if } & i \in I_{-}
\end{array}\right.
$$

Obviously we have

$$
K=\sum_{i \in I} \delta_{i} \cdot K_{i}
$$


and (3.4.1) holds. Furthermore, if $w=\sum_{i=1}^{k} \alpha_{i} \cdot u_{i}$, then $\left|\alpha_{i}\right| \leq(\text { Ind } K)^{-1 / k}$ and we have that

$$
\begin{aligned}
& \text { Ind } K_{i}=\frac{\left|u_{1} \wedge \cdots \wedge u_{i-1} \wedge w \wedge u_{i+1} \wedge \cdots \wedge u_{k}\right|}{\operatorname{det} \Lambda} \\
& =\frac{\left|\alpha_{i}\right| \cdot\left|u_{1} \wedge \cdots \wedge u_{k}\right|}{\operatorname{det} \Lambda} \\
& \leq(\operatorname{Ind} K)^{(k-1) / k}
\end{aligned}
$$

and therefore (3.4.2) holds. Finally, (3.4.3) holds since obviously

$$
\|w\| \leq\left\|u_{1}\right\|+\cdots+\left\|u_{k}\right\|
$$

Now we must show that the vector $w$ can be computed in polynomial time. Indeed, the problem of finding an integral nonzero vector in the parallelopiped $\Psi$ reduces to integer programming in fixed dimension $k$. Let us construct a basis $b_{1}, \ldots, b_{k}$ of the lattice $\Lambda$. This can be done in polynomial time (see [6]). We approximate the parallelopiped $\Psi$ by a rational parallelopiped $\Pi$. To do that, let us compute the basis $\left\{u_{i}^{*} \in \operatorname{Lin} K: i=1, \ldots, k\right\}$ of Lin $K$ conjugate to $\left\{u_{1}, \ldots, u_{k}\right\}$, so $\left\langle u_{i}, u_{j}^{*}\right\rangle=\delta_{i j}$ for $i, j=1, \ldots, k$. Let $D$ be the least common multiple of the denominators of coordinates of $u_{i}^{*}, i=1, \ldots, k$. We define the parallelopiped $\Pi$ replacing (Ind $K)^{-1 / k}$ in (3.4.4) by its rational approximation with error less than $1 / D$. This can be done in polynomial time as well. Then for $i=1, \ldots, k$ we solve a problem of integer linear programming:

$$
\begin{aligned}
& \text { Find } \gamma=\left(\gamma_{1}, \ldots, \gamma_{k}\right) \in \mathbb{Z}^{k} \\
& \text { Such that: } \sum_{i=1}^{k} \gamma_{i} \cdot b_{i} \in \Pi \\
& \text { and } \gamma_{i} \geq 1 .
\end{aligned}
$$

For some $i$ the program has a solution and then the point $w=\sum_{i=1}^{k} \gamma_{i} \cdot b_{i}$ is the desired nonzero integral vector from $\Psi$. Since $k$ is fixed, a solution of the above program can be found in polynomial time (see [6] and [7]).

Now we can prove the main result of this section.

(3.5) Theorem (see [2]). Let us fix $k \in \mathbb{N}$. Then, for any $d \in \mathbb{N}$, for any simple rational $k$-dimensional cone $K \subset \mathbb{R}^{d}$, a family of $k$-dimensional primitive cones $K_{i}$, $i \in I$, and integral numbers $\varepsilon_{i} \in\{1,-1\}, i \in I$, such that

$$
K=\sum_{i \in I} \varepsilon_{i} \cdot K_{i}
$$

can be constructed in polynomial time. 
Proof. We iterate the construction of Lemma 3.4. After the $m$ th iteration we get a decomposition

$$
K=\sum_{i \in I_{m}} \varepsilon_{i} \cdot K_{i}
$$

where \# $I_{m} \leq k^{m}$, Ind $K_{i} \leq(\text { Ind } K)^{((k-1) / k)^{m}}, \varepsilon_{i} \in\{-1,1\}$, and size $K_{i} \leq$ size $K+$ $O\left(d^{2} \cdot m\right)$. Let us choose the smallest integral $m$ such that

$$
m \geq \frac{-\log \log 1.9+\log \log (\operatorname{Ind} K)}{\log k-\log (k-1)} .
$$

Then after the $m$ th iteration we will have that Ind $K_{i} \leq 1.9$ and therefore Ind $K_{i}=1$ for all $i$ in the representation (3.5.1). Now we observe that the complexity of our algorithm is linear in $k^{m}$. We have that

$$
k^{m} \leq C_{1}(k) \cdot(\log (\operatorname{Ind} K))^{C_{2}(k)},
$$

where

$$
C_{1}(k)=\exp \left\{\left(\frac{-\log \log 1.9}{\log k-\log (k-1)}+1\right) \cdot \log k\right\}, \quad C_{2}(k)=\frac{\log k}{\log k-\log (k-1)} .
$$

Thus for a fixed $k$ the value of $k^{m}$ is bounded by a polynomial in the size of $K$.

\section{Constructing a Regular Subspace}

In this section we prove the following main result.

(4.1) Lemma. A polynomial-time algorithm exists which, for any given $d, k, m \in \mathbb{N}$ and any given $m$ primitive $k$-dimensional cones $K_{i} \subset \mathbb{R}^{d}, i=1, \ldots, m$, computes a $(d-k+1)$-dimensional subspace $G \in \mathbf{G}_{d-k+1}\left(\mathbb{R}^{d}\right)$ which is a regular point for all the functions $\mu\left(K_{i}\right), i=1, \ldots, m$.

We deduce Lemma 4.1 from a more general statement about polynomials defined by their oracles. Such an object is a multivariate rational polynomial $P$ given by a "black box" which for any given rational $x$ computes the value $P(x)$. Besides we assume that an upper bound $D$ of the degree of $P$ is known. This approach is useful when we deal with various determinants. Indeed, a determinant can usually be computed rather efficiently whereas its straightforward expansion contains plenty of monomial terms. The following result shows that we can always compute in polynomial time a point which is not a root of any polynomial from 
a given family, provided for each polynomial we know at least one point which is not a root. We note that generally it is an open problem to determine in polynomial time whether a polynomial given by an oracle is not identically zero.

(4.2) Proposition. For any finite set $\left\{P_{\alpha}: \alpha \in A\right\}$ of rational polynomials $P_{\alpha}: \mathbb{Q}^{n} \rightarrow \mathbb{Q}$ given by their oracles such that $\operatorname{deg} P_{\alpha} \leq D_{\alpha}, \alpha \in A$, and for any given $x_{\alpha} \in \mathbb{Q}^{n}, \alpha \in A$, such that $P_{\alpha}\left(x_{\alpha}\right) \neq 0$ for $\alpha \in A$ a vector $x \in \mathbb{Q}^{n}$ such that $P_{\alpha}(x) \neq 0$ for all $\alpha \in A$ can be computed in time which is polynomial in $n, \# A, \max \left\{D_{\alpha}: \alpha \in A\right\}$, and in the maximum size of $x_{\alpha}, \alpha \in A$.

Proof. Let us put $D=\max \left\{D_{\alpha}: \alpha \in A\right\}$. We construct an algebraic curve $\gamma: \mathbb{Q} \rightarrow \mathbb{Q}^{n}$ which passes through all the points $x_{\alpha}, \alpha \in A$. We may think of the elements of $A$ as the integers $1, \ldots, \# A$. Let us denote by $x_{\alpha}(j)$ the $j$ th coordinate of $x_{\alpha}$. Using standard interpolation we construct a polynomial $r_{j}(t)$, $t \in \mathbb{Q}, j=1, \ldots, n$, of degree $\# A-1$ such that $r_{j}(\alpha)=x_{\alpha}(j)$ for $\alpha \in A$. Put $\gamma(t)=$ $\left(r_{1}(t), \ldots, r_{n}(t)\right) \in \mathbb{Q}^{n}$. Then $\gamma(\alpha)=x_{\alpha}$ for $\alpha \in A$. We observe that for $\alpha \in A$ the polynomial $p_{\alpha}(t)=P_{\alpha}(\gamma(t))$ is a univariate nonzero polynomial of degree at most $(\# A) \cdot D_{\alpha}$. Our algorithm is the following: we compute consecutively $\gamma(t)$, $t=0, \ldots,(\# A)^{2} \cdot D$, and the corresponding values $P_{\alpha}(\gamma(t)), \alpha \in A$. For at least one $t=T \in\left[0:(\# A)^{2} \cdot D\right]$ all the values of $P_{\alpha}(\gamma(t)), \alpha \in A$, must be nonzero. We put $x=\gamma(T)$.

Now we can prove Lemma 4.1.

Proof of Lemma 4.1. We use (2.2) and (4.2). Let us define first the set of indices $A$. An index $\alpha \in A$ is a tuple $\alpha=(i, s)$, where $i=1, \ldots, m$ and $s=1, \ldots, k$. For $\alpha=(i, s)$ we construct the polynomial $P_{x}$ as follows. Choose the cone $K_{i}$. Construct an oriented basis $B_{s}=\left(b_{1}^{s}, \ldots, b_{d-k+1}^{s}\right)$ as described in (2.2). This can be done in polynomial time (see [6] for lattice algorithms). Then $P_{\alpha}$ will be a polynomial in the coordinates of $g_{1}, \ldots, g_{d-k+1} \in \mathbb{R}^{d}$ defined as $f_{s}$ (see (2.2)). We observe that for any given $g_{1}, \ldots, g_{d-k+1} \in \mathbb{R}^{d}$ the value of $P_{\alpha}$ can be computed using $O\left(d^{3}\right)$ arithmetic operations as the value of the $(d-k+1) \times(d-k+1)$ determinant. We also note that $\operatorname{deg} P_{\alpha} \leq d-k+1$. Our aim is to find a set of vectors $G=\left(g_{1}, \ldots, g_{d-k+1}\right)$ such that $P_{\alpha}(G) \neq 0$ for all $\alpha \in A$. To apply Proposition 4.2 we only need to construct a point $x_{a}$ such that $P_{\alpha}\left(x_{\alpha}\right) \neq 0$. However, we can choose $x_{\alpha}=\left(b_{1}^{s}, \ldots, b_{d-k+1}^{s}\right)$. Indeed, the value of $P_{\alpha}\left(x_{\alpha}\right)$ is the determinant of the Gramm matrix of the basis $B_{s}$, and therefore is nonzero.

\section{Proof of the Main Result}

Now we are ready to prove the main result of this paper.

Proof of Theorem 1.2. Our algorithm is the following. For each collection $I \subset[1: m]: \# I=k$ of indices, by applying the Volume Computation Oracle we check if $F_{I}=\left\{x \in P:\left\langle l_{i}, x\right\rangle=a_{i}, i \in I\right\}$ is a $(d-k)$-dimensional face of $P$ and if 
so, we compute its volume. Then for each such a face $F_{I}$ we find a set

$$
J_{I}=\left\{i \in[1: m]: \text { the face } F_{I} \text { belongs to the hyperplane }\left\langle l_{i}, x\right\rangle=a_{i}\right\}
$$

This can be done using linear programming or by applying the Oracle. For each face $F_{I}$ let us put $K_{I}=\operatorname{co}\left\{l_{i}: i \in J_{I}\right\}$. Thus $K_{I}$ is a rational $k$-dimensional polyhedral cone in $\mathbb{R}^{d}$. Then, using triangulation and Theorem 3.5 , we compute in polynomial time a decomposition

$$
K_{I}=\sum_{\alpha \in A_{I}} \varepsilon_{\alpha} \cdot K_{\alpha}
$$

where $K_{\alpha}, \alpha \in A=\bigcup A_{I}$, is a primitive $k$-dimensional cone in $\mathbb{R}^{d}$. Using Lemma 4.1 we compute a $(d-k+1)$-dimensional subspace $G$ which is a regular point for all the functions $\mu\left(K_{a}\right), \alpha \in A$. Then for all $\alpha \in A$ we compute the value $\mu_{G}\left(K_{\alpha}\right)$ as in (2.2), see also (2.1.2). Now we put

$$
\mu_{G}\left(K_{t}\right)=\sum_{\alpha \in A_{I}} \varepsilon_{\alpha} \cdot \mu_{G}\left(K_{\alpha}\right)
$$

Finally, we compute the coefficient $e_{d-k}(P)$ using Theorem 2.5.

Now we discuss the problem of counting integral points in a $d$-dimensional integral simplex. Let $\Delta \subset \mathbb{R}^{d}$ be a $d$-dimensional integral simplex. Our approach gives the following complexity for the problem of counting integral points in $\Delta$. Obviously, we have

$$
\#\left(\Delta \cap \mathbb{Z}^{d}\right)=\sum_{j=0}^{d} e_{j}(\Delta) .
$$

To compute $e_{j}(\Delta)$ we use our algorithm summarized in Theorem 1.2. We note that the volume of a face of $\Delta$ can be computed in polynomial time and that we have $2^{d+1}$ faces, including the empty face. The complexity of the algorithm from Lemma 3.4 is dominated by the term $(d \cdot \operatorname{size} \Delta)^{o(d)}$ for integer programming (see, for example, [7]). Thus the complexity of the algorithm from Theorem 3.5 is dominated by $(d \cdot \operatorname{size} \Delta)^{O(d)}$ too, because of the estimates on the number of iterations. Summarizing, we conclude that our algorithm for counting integral points in a simplex has $(d \cdot \operatorname{size} \Delta)^{O(d)}$ complexity. This is probably the best-known estimate so far.

\section{Acknowledgment}

I am grateful to Robert Morelli for many useful conversations. 


\section{References}

1. A. Barvinok, Computing the Ehrhart polynomial of a convex lattice polytope, Preprint, TRITA/ MAT-92-0036, Royal Institute of Technology, Stockholm, 1992.

2. A. I. Barvinok, A polynomial-time algorithm for counting integral points in polyhedra when the dimension is fixed, Proceedings of 34 th Symposium on the Foundations of Computer Science (FOCS '93), IEEE Computer Society Press, New York, 1993, pp. 566-572.

3. W. Cook, M. Hartmann, R. Kannan, and C. McDiarmid, On integer points in polyhedra, Combinatorica, 12 (1992), 27-37.

4. M. Dyer and A. M. Frieze, On the complexity of computing the volume of a polyhedron, SIAM J. Comput., 17(5) (1988), 967-974.

5. W. Fulton, Introduction to Toric Varieties, Annals of Mathematics Studies, Vol. 131, Princeton University Press, Princeton, NJ, 1993.

6. M. Grötschel, L. Lovasz, and A. Schrijver, Geometric Algorithms and Combinatorial Optimization, Algorithms and Combinatorics, Vol. 2, Springer-Verlag, Berlin, 1988.

7. R. Kannan, Minkowski's convex body theorem and integer programming, Math. Oper. Res., 12 (1987), 415-440.

8. J. Lawrence, Polytope volume computation, Math. Comp., 57(195) (1991), 259-271.

9. I. G. Macdonald, Polynomials associated with finite cell complexes, J. London Math. Soc. (2), 4 (1971), 181-192.

10. R. Morelli, Pick's theorem and the Todd class of a toric variety, Adv. in Math., 100(2) (1993), 183-231.

11. J. E. Pommersheim, Toric varieties, lattice points and Dedekind sums, Math. Ann., 295 (1993), 1-24.

12. R. P. Stanley, Enumerative Combinatorics, Vol. 1, Wadsworth \& Brooks/Cole, Monterey, CA, 1986.

Received November 3, 1992, and in revised form September 8, 1993. 